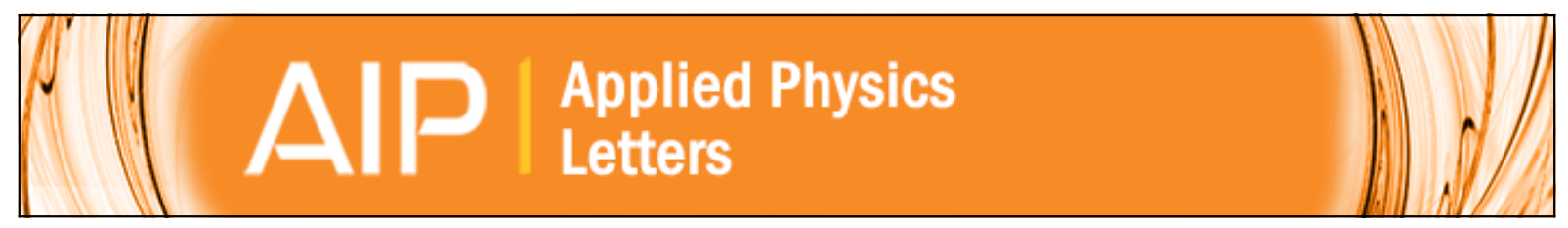

\title{
Charge carrier mobility in an organic-inorganic hybrid nanocomposite
}

Kaushik Roy Choudhury, Jeffrey G. Winiarz, Marek Samoc, and Paras N. Prasad

Citation: Applied Physics Letters 82, 406 (2003); doi: 10.1063/1.1537054

View online: http://dx.doi.org/10.1063/1.1537054

View Table of Contents: http://scitation.aip.org/content/aip/journal/apl/82/3?ver=pdfcov

Published by the AIP Publishing

\section{Articles you may be interested in}

Organic/inorganic hybrid pn-junction between copper phthalocyanine and CdSe quantum dot layers as solar cells J. Appl. Phys. 112, 044507 (2012); 10.1063/1.4747835

Charge transport in two different conductive polymer and semiconducting quantum dot nanocomposite systems J. Appl. Phys. 111, 044313 (2012); 10.1063/1.3682106

Enhanced photorefractive effect in liquid crystal structures co-doped with semiconductor quantum dots and metallic nanoparticles

Appl. Phys. Lett. 99, 191109 (2011); $10.1063 / 1.3659485$

Hole transport mechanism in organic/inorganic hybrid system based on in-situ grown cadmium telluride nanocrystals in poly(3-hexylthiophene)

J. Appl. Phys. 109, 114509 (2011); 10.1063/1.3594647

Efficient energy transfer in layered hybrid organic/inorganic nanocomposites: A dual function of semiconductor nanocrystals

Appl. Phys. Lett. 96, 083109 (2010); 10.1063/1.3319838

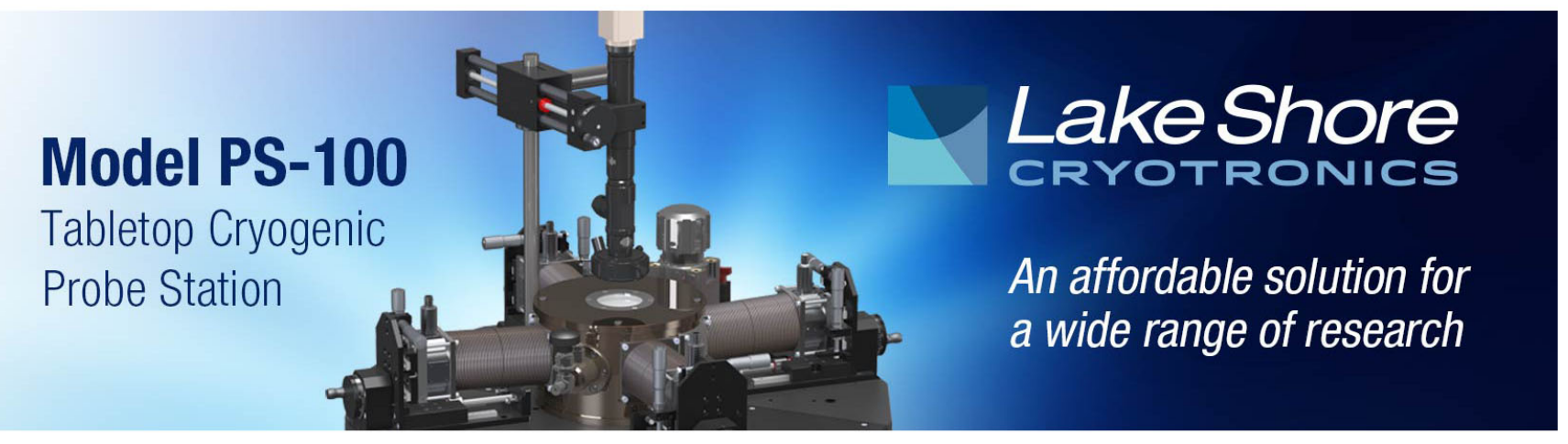




\title{
Charge carrier mobility in an organic-inorganic hybrid nanocomposite
}

\author{
Kaushik Roy Choudhury, Jeffrey G. Winiarz, Marek Samoc, ${ }^{\text {a) }}$ and Paras N. Prasad ${ }^{\text {b) }}$ \\ Institute for Lasers, Photonics and Biophotonics, Departments of Physics and Chemistry, State University \\ of New York at Buffalo, Buffalo, New York 14260
}

(Received 25 September 2002; accepted 21 November 2002)

\begin{abstract}
Organic-inorganic hybrid materials are media for electronic and optoelectronic applications. We present a study of the electronic transport in such a model nanoparticle-sensitized hybrid organic-inorganic photorefractive host system, consisting of poly(N-vinylcarbazole) doped with quantum dots of cadmium sulfide, using standard time-of-flight techniques. The photocurrent transients exhibit features typical of dispersive transport in an amorphous semiconductor. The hole mobility depends strongly on the electric field and temperature indicating Poole-Frenkel-like activated hopping transport; a thickness dependence of the mobility is observed. The presence of nanoparticles does not lead to increased trapping of holes. Conversely, a surprising result is observed: the mobility actually increases with the increase of nanoparticle concentration even though it is well below the percolation limit. (C) 2003 American Institute of Physics.
\end{abstract}

[DOI: $10.1063 / 1.1537054]$

Hybrid organic-inorganic materials have emerged as a class of electronic and optoelectronic media for a number of potential technological applications. Nanocomposites consisting of inorganic quantum dots and functional polymers have shown promise for photorefractive applications, lightemitting diodes, and photovoltaic devices. The advantages offered by these nanocomposites are tunability of the electronic and optical properties of the quantum dots with the functional and structural flexibility of a polymer. ${ }^{1-6}$ However, very little fundamental understanding of charge carrier transport in such hybrid materials exists. We present here the results of a charge carrier mobility study in a system comprised of cadmium sulfide nanoparticles (QCdS) dispersed in poly(N-vinylcarbazole) (PVK). Previous work has shown that doping PVK with nanoparticles is an effective method of sensitizing photorefractive composites. ${ }^{7,8}$ It has been demonstrated that through this approach several advantages are realized, perhaps the most attractive one concerns the ease with which the spectral properties of the quantum dots are modified. ${ }^{9,10}$ The tuning of the spectral properties of these nanocrystals is made possible by the fact that the magnitude of the optical band gap depends on the size of the nanocrystal. In addition to the modification of absorption spectra obtained through nanocrystal photosensitization, it appears that the photocharge generation quantum efficiency of a nanocomposite can be in excess of that obtainable by sensitization with organic dyes and exhibits a weaker electric field dependence. ${ }^{7,8,11}$

A frequently cited limitation of polymeric photorefractive materials is their relatively slow response time. The response time of photorefractive polymers is generally limited by the free charge-carrier mobility, $\mu$; thus, the knowledge of the influence of nanoparticle doping on charge transport in the composites becomes important for optimizing properties of such composites. Even among the molecularly doped

\footnotetext{
${ }^{a)}$ Present address: Laser Physics Centre, The Australian National University, Canberra, Australia.

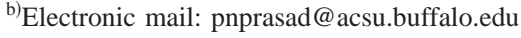

polymeric photorefractive materials, few studies have been conducted characterizing the charge transport properties, ${ }^{12}$ accentuating the need for such investigations.

The QCdS was synthesized using an emulsion method. ${ }^{13}$ The nanocrystal size was determined to be $<14 \mathrm{~nm}$ by means of x-ray diffraction and using the Scherrer formula; it was also confirmed by transmission electron microscopy. In the sample fabrication process, first a charge-generating layer of selenium $(<1 \mu \mathrm{m})$ was deposited by high vacuum deposition onto glass substrates precoated with indium tin oxide (Thin Film Devices). A solution of QCdS and PVK in pyridine was then spun onto the selenium coated indium tin oxide-glass substrate. The films were kept under vacuum for several hours to evaporate the solvent completely. The thickness of the films, as determined by an Alpha step profiler, ranged between 2.5 and $9 \mu \mathrm{m}$. Subsequently, silver counter electrodes were deposited.

The mobility of holes (dominant carriers in the PVK host materials) was measured using the conventional timeof-flight technique. ${ }^{14}$ Impurities contained within even carefully purified PVK inhibit the effective electron mobility within this matrix due to very strong trapping as was observed for this composite. The carriers were generated in the selenium layer by a $10 \mathrm{~ns}$ pulse from an $\mathrm{Nd}-\mathrm{yttrium}-$ aluminum-garnet laser at $532 \mathrm{~nm}$. Efficient photoinjection of holes occurring at the Se/PVK interface led to the appearance of hole transients attributable to transport in the polymer layer under the influence of applied bias. Most transients were measured using relatively high-intensity light pulses, since transit times were better defined under space-chargelimited conditions.

Figure 1 shows examples of photocurrent transients obtained for a $2 \%$ QCdS nanocomposite sample. It is evident that a "knee" is present in the transients indicating the arrival of the fastest photocarriers at the counter electrode. However, as usual in polymers or amorphous systems, there is also a pronounced "tail" of the signal due to slower car- 


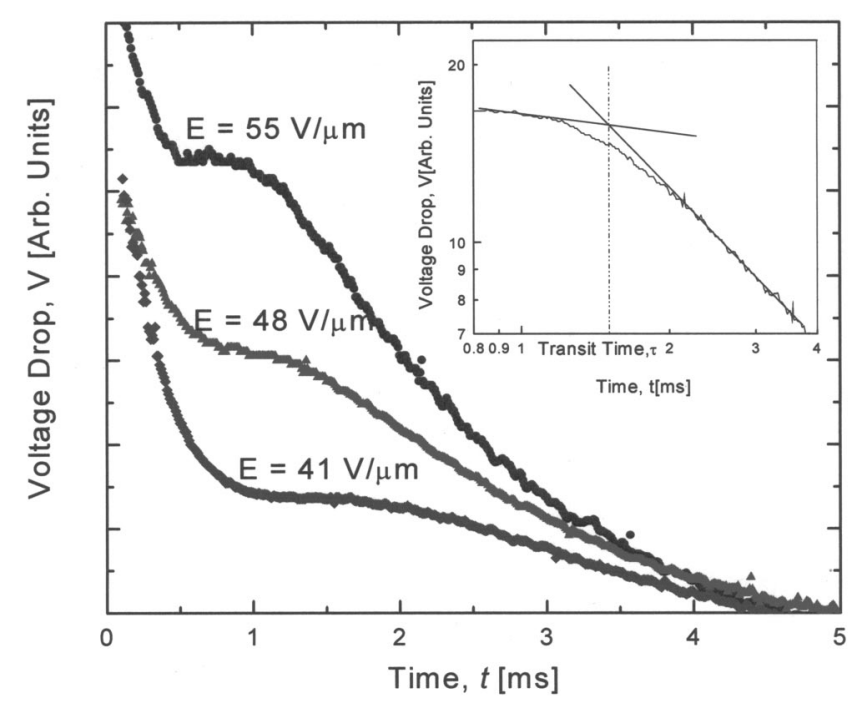

FIG. 1. Current transient curves for $2 \%$ CdS:PVK sample of $3.5 \mu \mathrm{m}$ thickness at $297 \mathrm{~K}$. Inset shows a typical trace on a $\log I-\log t$ plot used to determine transit time.

riers. Such dispersive transport is typically described in terms of the stochastic continuous-time-random-walk (CTRW) model developed by Scher and Montroll. ${ }^{15}$ This model assumes an amorphous state with large variations of distances between nearest-neighbor atoms or molecules, which results in a distribution, $\Psi(t)$ in the charge carrier hopping time, $t$, dictated by the equation $\Psi(t) \propto t^{-1-\alpha}$, where $\alpha$ is a dispersion parameter able to assume a value between 0 and 1 . Well-known consequences of such features of carrier transport are the "universality" of transient current traces $I$, observed in the pre- and post-transit time regimes, i.e., $I_{\text {pre }} \sim t^{-(1-\alpha)}, I_{\text {post }} \sim t^{-(1+\alpha)}$ and the electric field and sample thickness dependences of the effective mobility. Similar features of carrier transport can also be predicted from considerations of the kinetics of multiple trapping of charge carriers if a wide energetic distribution of trapping states is present. ${ }^{16-18}$

The dependence of the transit time on electric field, temperature, sample thickness, and concentration of the nanopar-

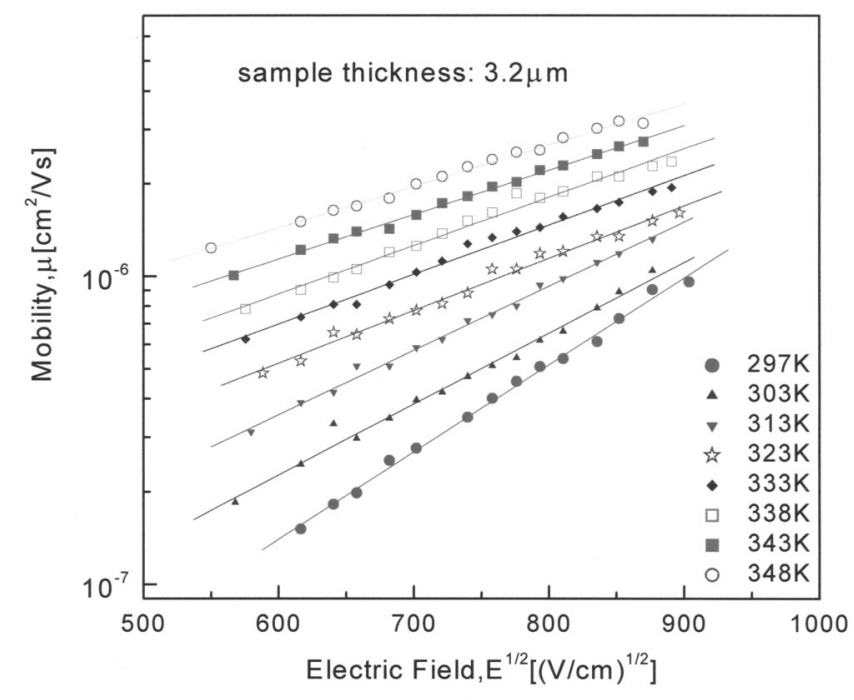

FIG. 2. Field dependence of the hole mobility in $2 \% \mathrm{CdS}$ :PVK sample parametric in temperature: the Poole-Frenkel behavior.

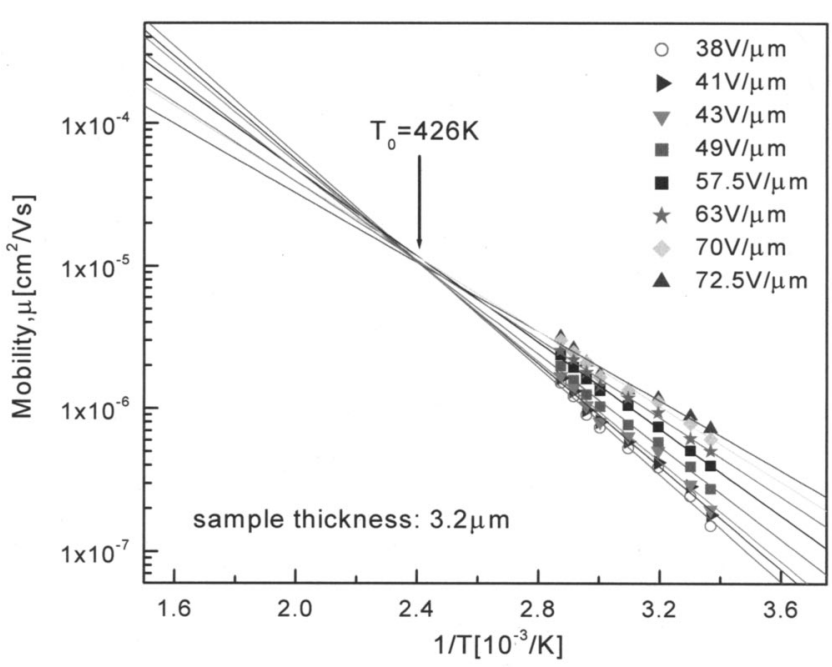

FIG. 3. Temperature dependence of hole mobility parametric in electric field plotted on an $\ln \mu$ vs $T^{-1}$ scale: the Arrhenius behavior.

ticles were studied and the results are presented in subsequent figures in the form of the effective mobility of charge carriers (defined as $\mu=L / t_{f} E$ ).

Figure 2 shows the field dependences of the mobility as measured at several temperatures for the same nanocomposite sample. To obtain a meaningful two-dimensional mapping of the temperature-field dependences it was imperative to perform the temperature and field cycling on the same sample, the thickness dependence of the effective mobility (vide infra) complicating comparisons between samples spun in different batches. The linear dependence on $E^{1 / 2}$ reveals the well-known Poole-Frenkel behavior characteristic for disordered materials. From the linear temperature dependences of the drift mobility for constant electric field in an Arrhenius plot (see Fig. 3) a field-dependent thermal activation energy can be invoked suggesting the application of Gill's phenomenological relation ${ }^{19}$

$\mu(E, T)=\mu_{0} \exp \left(-\frac{\Delta_{0}-\beta \sqrt{E}}{k_{B} T_{\text {eff }}}\right)$, where, $\frac{1}{T_{\text {eff }}}=\frac{1}{T}-\frac{1}{T_{0}}$

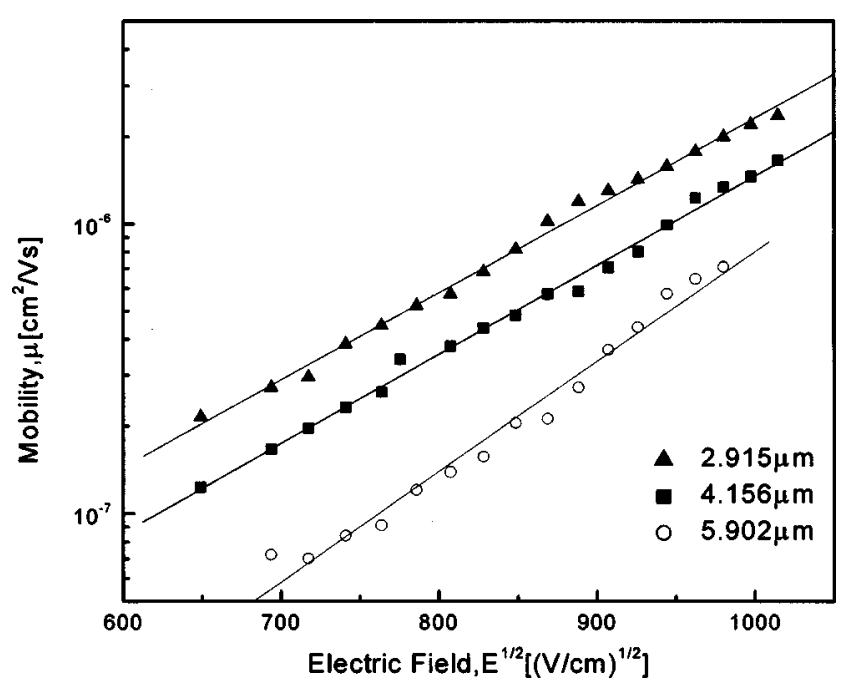

FIG. 4. Field dependence of mobility for $2 \% \mathrm{CdS} \cdot \mathrm{PVK}$ samples of different thickness at $297 \mathrm{~K}$. 


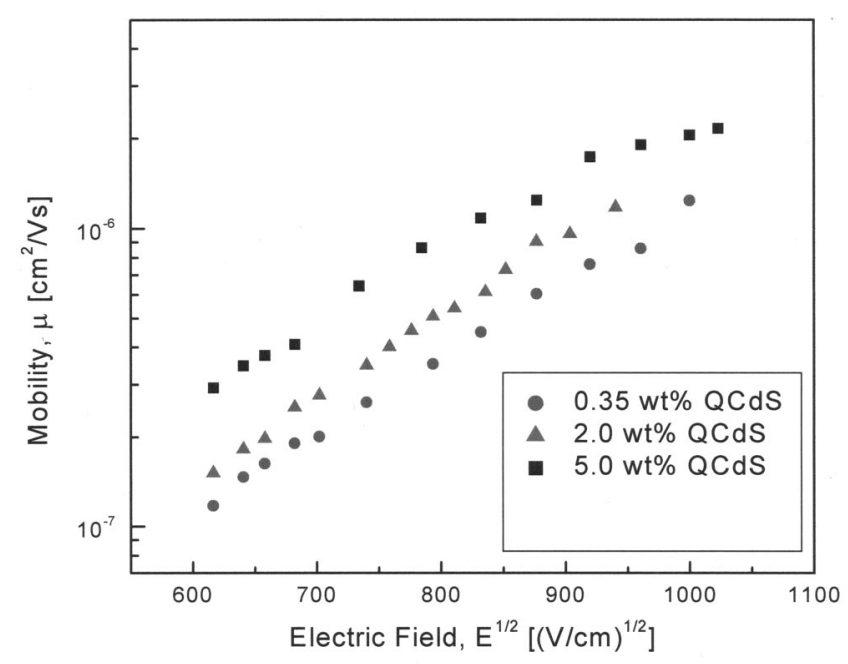

FIG. 5. Field dependence of the hole drift mobility for different concentration of CdS at $297 \mathrm{~K}$. The thicknesses of the samples were within a range of $0.3 \mu \mathrm{m}$ to eliminate thickness dependent effects as much as possible.

with $\Delta_{0}$ being the zero-field activation energy. The values of $\Delta_{0}$ and $\mu_{0}$ as determined from the data in Figs. 2 and 3 are $0.46 \mathrm{eV}$ and $1.08 \times 10^{-5} \mathrm{~cm}^{2} / \mathrm{V} \mathrm{s}$, respectively, and the parameter $T_{0}=426 \mathrm{~K}$. This, together with the range of mobility values obtained for all samples confirms the assertion ${ }^{1}$ that the hole transport in PVK composite samples is not very different from that in the host PVK polymer. ${ }^{14,20}$ The dispersive character of the transport is also evidenced by the sample thickness dependences shown in Fig. 4. The fact that the effective mobility values are higher in thinner samples is in qualitative agreement with predictions of theories of CTRW and of multiple trapping models and reflects the intuitive assertion that, as the sheet of charge carriers travels through the sample, longer trapping times or deeper local levels play a progressively more important role in determining the average velocity of the carriers.

The effect of nanoparticle concentration on the charge carrier mobility is shown in Fig. 5. One needs to be aware that relatively strong dependencies on the electric field and sample thickness must be reliably taken out of the comparisons to verify the concentration dependencies. Nevertheless, the trend seems to be quite unambiguous that samples with higher concentration of the nanoparticles also present higher hole mobilities.

This increase is surprising since the volume concentrations of the nanoparticles in this study are rather low (limited by the need for a good homogeneity of the sample), thus well below any percolation threshold. In the one-carrier transport situation (holes injected from a selenium electrode and not from the nanoparticles) the nanoparticle effect may be due to the modification of the field acting on carriers being transported through the polymer matrix or due to the actual participation of the QCdS in the carrier transport mechanism. The effect of the field modification (for example, through a Maxwell-Wagner type mechanism) should not be profound because of the relatively low volume fraction of the QCdS possessing the different dielectric constant. Another possibility of the mobility enhancement comes from a carrier tra- versing the sample actually encountering a nanoparticle on its way. For nanoparticles of diameter $d$ present in the sample at volume fraction $x$, their concentration is $N=6 x / \pi d^{3}$. With the cross section of an individual nanoparticle, $\sigma_{n}$, equal to $\pi d^{2} / 4$ the mean free path of a carrier between encountering a nanoparticle is $\bar{\ell}=1 / N \sigma_{n}=2 d / 3 x$, which for $15 \mathrm{~nm}$ particles present at a volume fraction of 0.01 would amount to $1000 \mathrm{~nm}$. Thus, a carrier has a chance of being captured (and released) by a nanoparticle several times while traversing a distance of a few micrometers. For a time- and thicknessdependent mobility (non-Markoffian transport) an important issue is if carriers injected back from a nanoparticle into the matrix will have their velocity enhanced compared to the average velocity of the rest of the population. The acceleration of the carriers by such encounters with nanoparticles can explain the observed increase in mobility. However, such a mechanism would also suggest a thickness independent mobility. Then, for the mobility in the polymer matrix showing a $\mu \propto L^{-\kappa}$ functional dependence, substitution of the mean free path for characteristic thickness would eventually lead to $\mu \propto x^{\kappa}$. But, a thickness dependence is present in our nanocomposite samples. Therefore, the enhancement of average mobility by encounters with nanoparticles may be only partial. Additional work is needed to fully establish the applicability of this mechanism.

This study was supported by a NSF, DMR Solid State and Polymer Chemistry Grant No. DMR0075867. Partial support by a Defense Research Initiative on Nanotechnology (DURINT), Contract No. F496200110358, through the Directorate of Chemistry and Life Sciences of the Air Force Office of Scientific Research is also acknowledged. The authors thank and acknowledge Dr. Amitava Patra for his active help in nanocrystal synthesis.

\footnotetext{
${ }^{1}$ Y. Wang and N. Herron, J. Lumin. 70, 48 (1996).

${ }^{2}$ K. Rajeshwar, N. R. de Tacconi, and C. R. Chenthamarakshan, Chem. Mater. 13, 2765 (2001).

${ }^{3}$ Y. Wang and N. Herron, Chem. Phys. Lett. 200, 71 (1992).

${ }^{4}$ Y. Wang, Pure Appl. Chem. 68, 1475 (1996).

${ }^{5}$ N. Herron and D. L. Thorn, Adv. Mater. 10, 1173 (1998).

${ }^{6}$ M. Lal, M. Joshi, D. N. Kumar, C. S. Friend, J. Winiarz, T. Asefa, K. Kim, and P. N. Prasad, Mater. Res. Soc. Symp. Proc. 519, 217 (1998).

${ }^{7}$ J. G. Winiarz, L. Zhang, J. Park, and P. N. Prasad, J. Phys. Chem. B 106, 967 (2002).

${ }^{8}$ J. G. Winiarz, L. Zhang, M. Lal, C. S. Friend, and P. N. Prasad, J. Am. Chem. Soc. 121, 5287 (1999).

${ }^{9}$ S. H. Wang, S. H. Yang, C. L. Yang, Z. Q. Li, J. N. Wang, and W. K. Ge, J. Phys. Chem. B 104, 11853 (2000).

${ }^{10}$ J. Nanda, K. S. Narayan, B. A. Kuruvilla, G. L. Murthy, and D. D. Sarma, Appl. Phys. Lett. 72, 1335 (1998).

${ }^{11}$ J. G. Winiarz, L. Zhang, M. Lal, C. S. Friend, and P. N. Prasad, Chem. Phys. 245, 417 (1999).

${ }^{12}$ U. Hofmann, M. Grasruck, A. Leopold, A. Schreiber, S. Schloter, C. Hohle, P. Strohriegl, D. Haarer, and S. J. Zilker, J. Phys. Chem. B 104, 3887 (2000).

${ }^{13}$ M. Chaterjee and A. Patra, J. Am. Ceram. Soc. 84, 1439 (2001).

${ }^{14} \mathrm{~J}$. Mort and D. M. Pai, Photoconductivity and Related Phenomena (Elsevier Scientific, Amsterdam, 1976).

${ }^{15}$ H. Scher and E. W. Montroll, Phys. Rev. B 12, 2455 (1975).

${ }^{16}$ J. Noolandi, Phys. Rev. B 16, 4466 (1977).

${ }^{17}$ J. Noolandi, Phys. Rev. B 16, 4474 (1977)

${ }^{18}$ F. W. Schmidlin, Phys. Rev. B 16, 2362 (1977).

${ }^{19}$ W. D. Gill, J. Appl. Phys. 43, 5033 (1972).

${ }^{20}$ G. Pfister, Phys. Rev. B 16, 3676 (1977).
} 